\title{
Thiamine: an efficient, biodegradable, green catalyst for one-pot synthesis of functionalized dihydropyridines
}

\author{
Hossein Ghafuri*, Negar Joorabchi, Moghadaseh Keshvari Kenari, Atefeh Emami \\ Catalysts and Organic Synthesis Research Laboratory, Department of Chemistry, Iran \\ University of Science and Technology, P.O. Box 16846-13114 Tehran, I. R. Iran. \\ E-mail: ghafuri@iust.ac.ir
}

\begin{abstract}
An efficient protocol was developed for the four component and one-pot synthesis of functionalized 1, 4-dihydropyridines by thiamine (VB1) as biocatalyst via the reaction of malononitrile, substituted aldehydes, dimethylacetylenedicarboxylate and substituted aniline in ethanol at room temperature. Availability and non-toxicity are from important advantages of this green catalyst. Also, easy work-up, no chromatographic purifications and high yields in short reaction time are othere benefites of this procedure.
\end{abstract}

Keywords: 1, 4-dihydropyridines, Thiamine, Biocatalyst

\section{Introduction}

Synthesis of various heterocyclic molecules from the readily available starting materials is from the main challenges in organic synthesis. Multicomponent reactions (MCRs), are the reactions which three or more reactants are combined together in the same pot to generate a product that incorporates substantial portions of all the reactants [1]. MCRs have some advantages, including simplicity, very short reaction times, high atomic economy and high selectivity. They are also useful synthetic tools and can be used as catalysts in the preparation of pharmaceutical heterocyclic compounds [2].

In medicinal chemistry, the synthesis of biologically active molecules is from important research fields. Heterocycles constitute the largest variety of organic molecules of chemical, biomedical and industrial significance [3]. Functionalized 1, 4-dihydropyridines are important compounds of biologically active heterocycles. Initially, these compounds were found to be calcium channel modulators, and were developed as cardiovascular and antihypertensive drugs, 
which include Feiodipine, Amlodipine and Nifedipine (Fig1) [2]. Also, they have different biological activities such as antioxidant, anticancer, and antitumor activity [1]. Regarding to importance of 1, 4 dihydropyridines the different methods have been developed to the synthesis of these compounds.

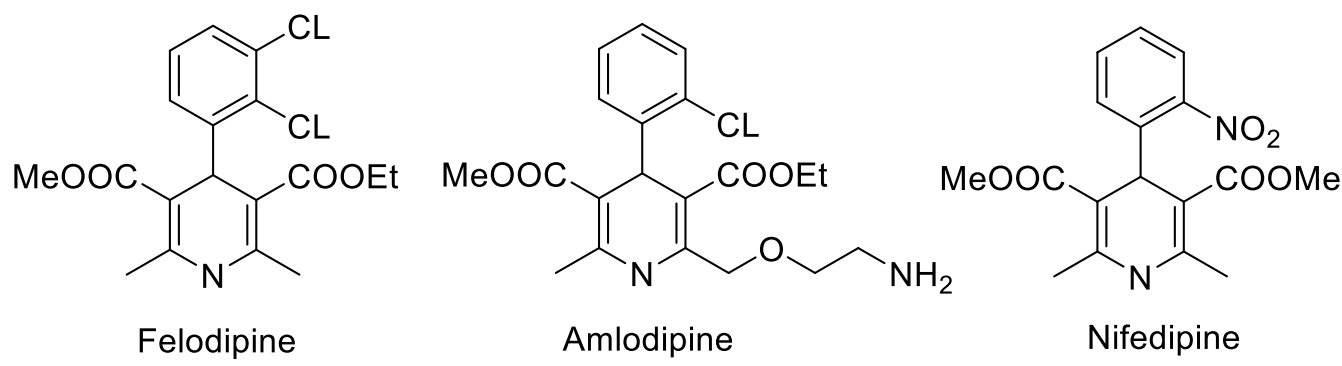

Fig. 1 some biologically important dihydropyridines

Thiamine (vitamin $\mathrm{B}_{1}-\mathrm{VB}_{1}$ ) is a member of the vitamin $\mathrm{B}$ complex group. Daily intake of thiamine is essential for healthy metabolism. Thiamine helps convert food into energy and maintain the nervous system. Vitamin B is a main factor for healthy functioning of all cells, especially nerve cells. Deficit of thiamine in a diet leads to a decline of the nervous system the so-called beriberisyndrome. Thiamine is widely found in nature. For example, $\mathrm{VB}_{1}$ can be found in high concentration in seeds, most notably rice and cereal grains. Thiamine is soluble in water and contains a primidine ring and a thiazole ringe linked by methylene bridge. The matter is an inexpensive and non-toxic reagent [4-5].

\section{Experimental}

General

All the solvents, chemical and reagents were purchased from Merk and Aldrich. Melting points were measured on an Electrothermal 9100 apparatus.

General procedure for the synthesis of 1, 4-dihydropyridines 5a-e

A solution of $140 \mathrm{mg}$ 4-chlorobenzaldehyde $(1.0 \mathrm{mmol}), 66 \mathrm{mg}$ malononitrile $(1.0 \mathrm{mmol})$, and thiamine $(0.1 \mathrm{mmol})$ were stirred in $1 \mathrm{ml}$ ethanol at room temperature. Then, a solution of 107 $\mathrm{mg}$ 4-methylaniline $(1.0 \mathrm{mmol})$ and $142 \mathrm{mg}$ dimethyl acetylenedicarboxylate $(1.0 \mathrm{mmol})$ in 2 $\mathrm{ml}$ ethanol was added. The resulting mixture was stirred until the reaction completed and the end of reaction indicated by thin-layer chromatography (TLC). The resulting precipitates were 
collected by filtration and washed with ethanol. The crude product was purified via recrystallization by ethanol.

\section{Results and discussion}

To optimize the reaction conditions, we conducted the reaction of 4-chlorobenzaldehyde, $\mathrm{p}$ tolouidine, malononitrile, and dimethyl acetylenedicarboxylate at room temperature in various conditions. As shown in Table 1, in the absence of catalyst, no product was formed. Some commercially available catalysts including $\mathrm{Al}_{2} \mathrm{O}_{3}$ and $\mathrm{Et}_{3} \mathrm{~N}$ were also tested, in which the product was obtained in low yields. Thiamine was found to be the most effective catalyst for this transformation since it gave the highest yield of product. The efficiency of the catalyst was found to be affected the quantity of the used catalyst in the reaction. We realized that the use of $10 \mathrm{mmol} \%$ thiamine is the best concenteration to promote the reaction (Table 1, entry 5).

Table 1 Influence of different catalysts for the synthesis of functionalized dihydropyridines ${ }^{\mathrm{a}}$

\begin{tabular}{|c|c|c|c|c|}
\hline Entry & Catalyst & $\begin{array}{c}\text { Catalyst Loading } \\
(\text { mol \%) }\end{array}$ & Time/h & $\begin{array}{l}\text { Yield } \\
(\%)^{b}\end{array}$ \\
\hline 1 & - & - & 10 & 0 \\
\hline 2 & $\mathrm{AL}_{2} \mathrm{O}_{3}$ & 10 & 10 & $10^{(2)}$ \\
\hline 3 & $\mathrm{Et}_{3} \mathrm{~N}$ & 10 & 10 & $15^{(2)}$ \\
\hline 4 & Thiamine & 10 & 1 & 80 \\
\hline 5 & Thiamine & 5 & 1 & 75 \\
\hline 6 & Thiamine & 15 & 1 & 80 \\
\hline
\end{tabular}

Then, the effect of solvents was evaluated and the results summarized in Table 2 . The results showed that the efficiency and yield of the reaction in ethanol were higher than those obtained in other solvent such as $\mathrm{H}_{2} \mathrm{O}$ or under solvent-free conditions.

Table 2 Optimization of reaction conditions

\begin{tabular}{cccc}
\hline Entry & Solvent & Time (h) & Yield (\%) \\
\hline $\mathbf{1}$ & No & 10 & 0 \\
$\mathbf{2}$ & $\mathrm{H}_{2} \mathrm{O}$ & 10 & 20 \\
$\mathbf{3}$ & ETOH & 1 & 80 \\
\hline${ }^{a}$ Yield refers to isolated pure product & &
\end{tabular}

${ }^{\text {a }}$ Yield refers to isolated pure product

To determine the efficiency of this multicomponent reaction, a series of different aldehydes were employed. As shown in Table 3, this protocol can be applied to aromatic aldehydes either with electron-withdrawing groups or electron-donating groups. Furthermore, a series of 
different substituted aldehydes gave excellent yields. It has been observed that the benzaldehyde with electron-withdrawing groups led to products in shorter reaction time. Unfortunately, when aliphatic aldehyde instead of aromatic aldehyde, after $24 \mathrm{~h}$, no reaction was observed. Aromatic amines with stronger electron-donor groups showed less reactivity, and more reaction time was required than electron-withdrawing substituents. As revealed in Table 3, these reactants worked well and furnished the desired products in high yields.

Table 3 Preparation of functionalized dihydropyridines catalyzed by Thiamine

\begin{tabular}{ccccccccc}
\hline \\
\end{tabular}

a Yield refers to isolated pure product.

To explain the mechanism of this multicomponent reaction, we proposed a plausible reaction course (Scheme 1). Knoevenagel condensation of aldehyde with malononitrile and thiamine as base catalyst yielded arylidene malononitrile (A). Arylamine was added to acetylenedicarboxylate to give the 1,3-dipole intermediate (B) and by a Michael addition between $\mathrm{A}$ and $\mathrm{B}$ the $\mathrm{C}$ intermediate mulecule was formed and the reaction, followed by migration of the hydrogen atom, intramolecular addition, and tautomerization to give D. 


\section{Scheme 1}<smiles>N#CC(C#N)=CC=O</smiles>

A

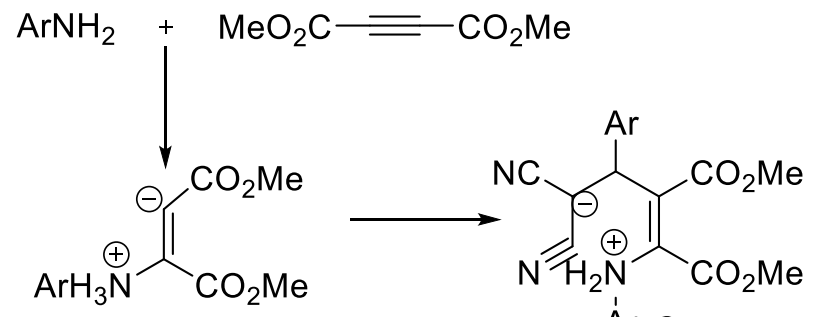

$B$<smiles>COC(=O)[Al]C1C(C#N)=C(N)N([Al])C(C(=O)OC)=C1C(=O)OC</smiles>

D

\section{Conclusions}

In conclusion, we have developed a facile and efficient one-pot, fourcomponent protocol for the synthesis of highly substituted dihydropyridines at room temperature in high yields. This domino reaction proceeded smoothly in good to excellent yields and offered several advantages including short reaction time, simple experimental procedure, and no toxic by-product.

\section{Acknowledgements}

The authors gratefully acknowledge the partical support from the Rrsearch Council of the Iran University of Science and Technology.

\section{References}

1. Pal, S.; Choudhury, L. H.; Parvin, T., Communication of Synthetic Organic Chemistry, 43, 986-992 (2013).

2. Kumar, A.; Sharma, S., Green Chemistry, 13, 2017-2020 (2011).

3. Chen, H. S.; Guo, R. Y., Monatshefte fur Chemie, 146, 1355-1362 (2015).

4. Mandhane, P. G.; Joshi, R. S.; Nagargoje, D. R.; Gill, C. H., Chinese Chemical Letters, 22, 563-566 (2011).

5. Sharma, P.; Gupta, M., Journal of Chemical Sciences, 128, 61-65 (2016). 